\title{
Personalidad de marca de los partidos políticos en Chile: una mirada desde los ciudadanos más jóvenes
}

\author{
Brand personality of political parties in Chile: a view from the \\ youngest citizens
}

\author{
Personalidade de marca dos partidos políticos no Chile: um olhar desde os \\ cidadãos mais jovens
}

RODRIGO URIBE, Universidad de Chile, Santiago, Chile (ruribe@fen.uchile.cl)

CRISTIAN BUZETA, Universidad de Chile, Santiago, Chile (cbuzetar@fen.uchile.cl]

JAVIERA REYES, Universidad de Chile, Santiago, Chile (jareyes@fen.uchile.cl)

\section{RESUMEN}

Este estudio examina la adecuación de la noción de personalidad de marca (Aaker, 1997) en el ámbito de los partidos políticos chilenos, midiendo su percepción en jóvenes universitarios. Se muestra que este concepto posee una validez de constructo y confiabilidad adecuada, observándose una estructura de cinco dimensiones (honestidad, capacidad, ser actual, energía y ser diferente) similar a la reportada en estudios previos (Smith, 2009). Adicionalmente, se presenta un análisis exploratorio de tres partidos políticos (izquierda, centro y derecha), encontrando percepciones deficientes de estos, con diferencias relevantes según autoposicionamiento político y grupo socioeconómico de los entrevistados.

Palabras clave: marketing político; comunicación política; partidos políticos; branding.

\section{ABSTRACT}

This study examines the adequacy of the notion of brand personality (Aaker, 1997) in the field of Chilean political parties, measuring their perception in a sample of university students. It is shown that this concept has an adequate validity of construct and reliability, observing a structure of five dimensions (honesty, capacity, up-to-date, energy and uniqueness) similar to that reported in previous studies (Smith, 2009). Additionally, an exploratory analysis of three political parties (left, center and right) is presented, finding deficient perceptions of these, with relevant differences according to the political self- positioning and socioeconomic level of the interviewees.

Keywords: political marketing; political communication; political parties; branding.

\section{RESUMO}

O presente estudo examina a adequação da noção de personalidade da marca (Aaker, 1997) nos partidos políticos chilenos, medindo sua percepção em jovens estudantes universitários. Mostra-se que esse conceito possui validade na construção e confiabilidade, além de se observar uma estrutura de cinco dimensões (honestidade, capacidade, ser atual, energia e ser diferente) semelhante à descrita em estudos anteriores (Smith, 2009). Além disso, é apresentada uma análise exploratória de três partidos políticos (esquerda, centro e direito), encontrando percepções deficientes sobre esses partidos, com diferenças relevantes de acordo com o auto posicionamento político e o grupo socioeconômico dos entrevistados.

Palavras-chave: marketing político; comunicação política; partidos políticos; branding.

Forma de citar:

Uribe, R., Buzeta, C. \& Reyes, J. [2017) . Personalidad de marca de los partidos políticos en Chile: una mirada desde los ciudadanos más jóvenes. Cuadernos.info, (41), 89-104. https://doi.org/10.7764/cdi.41.1252 


\section{INTRODUCCIÓN}

A pesar de que el uso de técnicas de marketing tiene larga data en la acción política, el marketing político como área de estudio es relativamente nuevo. Su desarrollo como disciplina se inició en la década de los setenta, buscando aplicar las estrategias desarrolladas en contextos comerciales a la acción política, sobre todo tratando de incrementar la efectividad de las campañas en el electorado (O'Shaughnessy, 1990).

Resulta llamativo que, aunque el marketing como disciplina abarca elementos que van más allá de la acción publicitaria, la gran mayoría de los trabajos en política se ha concentrado en esta última área. Un claro ejemplo de este déficit de investigación es el branding político, área íntimamente ligada a la comunicación, cuyo foco es la administración de una imagen positiva y diferenciada de candidatos y partidos (como si fueran marcas), buscando que ello actúe como motor de preferencia por parte de la ciudadanía (Newman, 1999).

Un concepto central en el branding es el de personalidad de marca, noción definida por Aaker (1997) y que plantea que las personas atribuimos un conjunto de características humanas a las marcas, las que actúan como base de la relación simbólica que establecemos con ellas. Lo relevante de lo anterior es que las investigaciones en el ámbito comercial han tendido a mostrar que este concepto es un predictor fundamental de la disposición y preferencia efectiva hacia productos o servicios (Ahmad \& Thyagaraj, 2015).

Este estudio examina la adecuación de la noción de personalidad de marca en su definición original al ámbito de la percepción de los partidos políticos. Se busca identificar la validez de su uso y las dimensiones clave de personalidad de marca que actúan en este caso, estableciendo adicionalmente en qué medida corresponden a los mismos rasgos reportados previamente por la investigación original en el ámbito de marcas comerciales. Antecedentes de lo anterior se encuentran en pocos estudios, siendo el más importante el desarrollado por Smith (2009), que estableció la utilidad y dimensiones de este concepto en el contexto de partidos políticos británicos

Adicionalmente, este estudio se desarrolló en una muestra de estudiantes universitarios, que forman parte del segmento de jóvenes entre 18 a 25 años, identificado como uno de los grupos en que más fuertemente se manifiesta el desprestigio de la política (Maureira, 2008). En efecto, es en este segmento etario en donde se ha detectado que, independientemente del grado de politización de las personas y de cómo se articule su discurso sobre la política, todos convergen en un espacio común: el rechazo a las instituciones políticas formales (Sandoval \& Carvallo, 2017).

\section{MARCO TEÓRICO}

BRANDING Y PERSONALIDAD DE MARCA

Una marca se define como un nombre distintivo o símbolo capaz de identificar y representar a un producto o servicio ofrecido por una organización, generando una diferencia en un mercado (Keller, 2003). En ese sentido, la marca es un activo que debe ser gestionado de modo tal que se perciba dicha diferenciación y que esta genere en los usuarios recuerdo, apego emocional y conducta de compra (Peng \& Hackley, 2009).

Para el logro de lo anterior es fundamental entender cuáles son las dinámicas principales de los consumidores en su percepción de las marcas. Una noción central, en este sentido, es la personalidad de marca, concepto que da cuenta de cuáles son las dimensiones clave que los consumidores emplean para interpretar las acciones de las marcas y desarrollar preferencia por estas (Aaker, 1997). En esta noción se asume que a través del contacto directo e indirecto con las marcas las personas perciben atributos funcionales (precio, sabor, duración, entre otras) y emocionales (cercana, juvenil, familiar, etcétera), los cuales son simbólicamente entendidos como un modo de ser de las marcas. De este modo se produce una antropormorfización de estas (Guthrie, 1997) que sienta las bases de la relación consumidor-marca, condicionando de modo significativo la disposición y preferencia o no preferencia en un mercado (Murphy, Moscardo, \& Benckendorff, 2007).

\section{LA MEDICIÓN DE LA PERSONALIDAD DE MARCA}

En su trabajo seminal, Aaker (1997) sistematiza el concepto de personalidad de marca y plantea un modelo de cinco dimensiones clave que los consumidores emplean para evaluarlas: sinceridad, emoción, competencia, sofisticación y rudeza (véase la tabla 6 al final del texto). A través de un estudio en tres etapas, que comenzó por una exhaustiva revisión bibliográfica, construyó un listado de más de 300 características o rasgos de personalidad. Luego, evaluó la relevancia y adecuación de estos rasgos con base en cuestionarios con consumidores, llegando a un conjunto tentativo de 114 rasgos. Finalmente, desarrolló un análisis factorial con el que redujo a un listado de 42 ítems (rasgos), conformando su instrumento final.

Estudios posteriores realizados fuera de los Estados 
Unidos han revisado la validez y confiabilidad de la estructura de dimensiones propuesta por Aaker (1997), examinando las potenciales variaciones culturales, así como por tipo de tipo de producto que pudiera presentar. Olavarrieta, Friedmann y Manzur (2010) validaron el uso del modelo de personalidad de marca original aplicándolo a 37 marcas chilenas representativas de diferentes industrias. Estos autores comprobaron la dimensionalidad del modelo (obteniendo una varianza explicada de casi 80\%) con la existencia de seis dimensiones: las mismas propuestas por Aaker, a las que se agrega la de tradición. Farrandi, Valette-Florence, $\&$ Fine-Falcy (2000) evaluaron su validez en el contexto francés, reduciendo el número de rasgos a 33, pero obteniendo la misma estructura original de cinco dimensiones de Aaker (1997). Por su parte, Austin, Siguaw y Mattila (2003) analizaron esta escala en el contexto de marcas de restaurantes. Los resultados muestran que la estructura de factores (dimensiones) de Aaker poseía indicadores adecuados de validez para el análisis de la categoría en general (todas las marcas conjuntamente), pero se encontraron algunas diferencias en su aplicación a marcas individuales. Así, más allá de algunas limitaciones reportadas y de las variaciones observadas en contextos diferentes, la escala de Aaker (1997) sigue siendo la más robusta y usada en la literatura, principalmente porque provee una herramienta con niveles de validez altos, con escalas confiables y que además es relativamente sencilla de emplear (Lee \& Cho, 2012).

\section{PARTIDOS POLIITICOS: MARCAS Y PERSONALIDAD DE MARCA}

No existe consenso en aceptar que los partidos puedan ser tratados propiamente como marcas. El principal argumento que se ha dado es que el mundo político es-en esencia-diferente de los mercados comerciales (Rothschild, 1979). Se ha sostenido la diversa naturaleza de los productos y servicios respecto de la actividad política, así como de la decisión a la que se ven enfrentados consumidores versus votantes (Lock \& Harris, 1996).

Sin desconocer dichas diferencias, otros autores han destacado que las personas, en sus diversos roles (como consumidores y ciudadanos), se relacionan con nombres, términos o símbolos (es decir, marcas), que identifican a ciertos grupos, organizaciones, servicios o productos, y que esos nombres son un factor clave en el proceso decisorio de desarrollar preferencia (Newman, 1999). Con esto, han sostenido que los partidos políticos se ajustan en términos generales al proceso de análisis de marca, como denominaciones (nombres) que generan una representación mental que actúa como uno de los fundamentos centrales del proceso decisorio político (Smith, 2009).

Así, la personalidad de marca de partidos políticos ha sido definida como una red de asociaciones de características humanas relativas a una agrupación política, que están en la memoria y a las que se puede acceder cuando se estimula el recuerdo o se realiza un llamado a la acción de un votante (Smith, 2009). Tal como ocurre en los mercados, la construcción de la personalidad de marca de un partido ocurre mediante el contacto directo o indirecto que cotidianamente las personas tienen con los partidos, lo cual ocurre fundamentalmente a través de espacios comunicacionales (es decir, noticias, campañas políticas). En este proceso, son atribuidos a los partidos ciertos rasgos derivados de la imagen que se va construyendo perceptualmente de estos (Gorbaniuk, Kusak, Kogut, \& Kustos, 2015). Eso no significa, eso sí, que la personalidad de marca de un partido sea el único determinante de la intencionalidad de voto o de la adhesión a un partido. Junto a ella, existen otros componentes fundamentales de esta disposición, como la simpatía política previa, el menú de medios consumidos, elementos de la campaña y las características de los candidatos (Banerjee \& Chauduri, 2016).

En el caso particular de los partidos políticos, solo tres estudios indexados en la Web of Science (WoS) han examinado la personalidad de marca, dos de ellos usando el modelo de Aaker (1997). Schneider (2004) analizó la personalidad de marca de políticos y partidos alemanes usando los rasgos originales descritos. Si bien este autor logró detectar que estos rasgos son capaces de diferenciar a los diversos actores y entidades políticas, no examinó las propiedades psicométricas de las mediciones realizadas, por lo que no existe un análisis de la dimensionalidad de las características de personalidad (Gorbaniuk et al., 2015)

En segundo término, el ya mencionado estudio de Smith (2009) es sin duda el más sólido de los artículos desarrollados a la fecha. Dicho trabajo examinó el concepto de personalidad de marca original de Aaker en partidos políticos usando una muestra de 183 estudiantes universitarios ingleses. En la fase inicial del estudio, se pidió a un grupo de expertos evaluar los rasgos, eliminándose ocho de la lista original de Aaker (1997) por considerarse ajenos a la política. Luego de este proceso, se aplicó a una muestra de estudiantes el instrumento con 34 rasgos, pidiendo evaluar con 


\begin{tabular}{ll} 
Dimensión & Rasgos \\
\hline Sinceridad (Honesty) & Honesto, cercano, íntegro, sincero, real, sentimental, realista y amistoso. \\
\hline Energético (Spirited) & Animado, osado, imaginativo, moderno y alegre. \\
\hline Imagen (Image) & Tranquilo, atractivo, a la moda, joven, cool, emocionante y actual. \\
\hline Liderazgo (Leadership) & Líder, confiable, inteligente, exitoso, trabajador, profesional y seguro. \\
\hline Rudeza (Thoughness) & Masculino, fuerte, resistente y extrovertido. \\
\hline Singularidad (Uniqness) & Único, independiente y original.
\end{tabular}

Tabla 1. Dimensiones de personalidad de marca de los partidos políticos

Fuente: Elaboración propia con base en Smith (2009).

base en ellos al partido laborista y el conservador. Los resultados develaron una estructura dimensional para los partidos británicos levemente diferente respecto de aquellas que tradicionalmente se han encontrado para las marcas en general. Se observó la existencia de seis dimensiones (en lugar de cinco): sinceridad, energético, imagen, liderazgo, rudeza y singularidad (tabla 1).

Un tercer estudio fue recientemente presentado por Gorbaniuk et al. (2015). Dichos autores realizaron un análisis en Polonia con una lista de rasgos distinta a la de Aaker (1997). Con base en entrevistas con 120 personas, extrajeron más de 3200 asociaciones posibles con partidos, las que fueron subsecuentemente sometidas a una evaluación de frecuencia, quedando 102 descriptores o rasgos de personalidad. A partir de eso, los autores desarrollaron la fase final que les permitió obtener una estructura dimensional de tres factores: integridad, desagrado y fortaleza. Finalmente, desarrollaron un nuevo estudio que confirmó esta estructura factorial, evaluando además las propiedades psicométricas (dimensionalidad y confiabilidad) de la escala usada.

A lo anterior se agregan dos estudios indexados en Scopus/SciELO ${ }^{1}$ desarrollados por Araya-Castillo y Etchebarne (2014a; 2014b) en Chile. Estos autores buscaron desarrollar un modelo propio de personalidad de marca de partidos (similar al trabajo de Gorbaniuk et al., 2015) basados en entrevistas con jóvenes universitarios. Luego de una fase exploratoria, estos académicos llegaron a un modelo de ocho factores: sinceridad, ambición, competencia, estimulante, tradicional, sofisticado, rudeza y elitista (Araya-Castillo \& Etchebarne, 2014a). En una segunda entrega, este modelo fue aplicado a partidos chilenos (Araya-Castillo \& Etchebarne, 2014b), observándose que aquellos de derecha aparecen más frecuentemente asociados a los atributos de ser conservadores, formales, tradicionales y ambiciosos, los de centro como más oportunistas, conflictivos y también ambiciosos, y los de izquierda más agresivos y apasionados. Una de las dificultades con este análisis es que no reporta valores obtenidos ni las diferencias estadísticas entre los partidos. Por lo tanto, los resultados quedan más bien como una descripción de atributos más presentes en cada partido, pero no se sabe si efectivamente se debe a que poseen o no puntajes significativamente superiores en esa dimensión.

\section{METODOLOGÍA}

Durante el primer semestre del año 2017, se realizó un estudio cuantitativo transversal, con base en encuestas aplicadas a una muestra de 276 estudiantes universitarios entre 18 y 25 años. Este grupo de edad cumple con ser parte de una generación que compone un porcentaje importante de las cifras de abstención en política². No obstante, es fundamental aclarar que se trata de una muestra con niveles de educación muy superiores al promedio de la población chilena en general, y de esa edad en particular. El uso de esta muestra responde a criterios tanto de accesibilidad, de representar al grupo que ha sido frecuentemente usado en los estudios previos, como de ser personas que poseen características sociodemográficas relativamente homogéneas en términos de educación como sociocultural (permitiendo disminuir la influencia de otras variables exógenas)

\section{VARIABLE DEPENDIENTE}

a. Percepción de personalidad de marca de partidos políticos: se utilizó la escala de Smith (2009), 
que a su vez es una adaptación de la escala de Aaker (1997), que representa la de mayor uso y robustez en la actualidad. Para ello, se evaluaron 34 rasgos a través de una escala Likert, de 1 a 5 (tabla 2). A las personas se les solicitó que respondieran a la siguiente pregunta para cada partido analizado: en una escala de 1 a 5, donde 1 es nada y 5 es totalmente, ¿̇en qué medida cada una de las siguientes características describen en su percepción del partido...? Se incorporaron tres partidos políticos, uno de derecha (Unión Demócrata Independiente-UDI), uno de centro (Democracia Cristiana-DC) y uno de izquierda (Partido Comunista-PC). Estos partidos fueron escogidos por representar diversas posiciones del espectro, por ser reconocidamente identificados con esas respectivas tendencias y por ser partidos nacionales en los últimos 10 años (SERVEL, 2015). La evaluación de los partidos se realizó en orden aleatorio para evitar efectos de sesgo de orden (Malhotra, 2008)

\section{VARIABLES INDEPENDIENTES}

a. Sexo: se midió con una pregunta directa en la cual el individuo se autodefine como hombre o mujer.

b. Grupo socioeconómico (GSE): se midió mediante una escala abreviada desarrollada por Kantar IBOPE Media (2016): consiste en tres ítems que concluyen en una aproximación del nivel socioeconómico con base en preguntas sobre el principal sostenedor del hogar (nivel educacional y jerarquía ocupacional) y la tenencia de once bienes y servicios. A partir de ello, se construye un puntaje que permite clasificar a las personas según su nivel socioeconómico.

c. Auto posicionamiento político: se midió a través de una pregunta en las que los individuos se situaron en un continuo de posición política. Se usó una escala de 1 a 10, donde 1 representa extrema izquierda y 10 extrema derecha (Frías, 2001).

\section{PROCEDIMIENTO}

a. Pre-test: se realizó un estudio previo buscando realizar una primera revisión de la escala. Comenzó con una validación de expertos, pidiendo a un grupo de cuatro especialistas en estudios de opinión pública que establecieran la pertinencia de los rasgos definidos por Smith (2009) en su versión en español -construida con base en la traducción de Olavarrieta et al. (2010) de los rasgos de Aaker (1997)-. No fue eliminado ningún ítem luego de esta revisión. La segunda parte del pre-test se realizó durante el mes de mayo de 2017, aplicándose el cuestionario a una muestra análoga a la usada en la fase final del estudio ( $n=24)$. En esta etapa, se verificó la duración promedio de la encuesta (siete minutos) y la compresión de los ítems/rasgos por parte de los entrevistados.

b. Desarrollo del estudio: una vez realizada la validación preliminar de la escala propuesta, se realizó la toma de datos con la muestra final. Se llevó a cabo una encuesta presencial durante el mes de junio de 2017, obteniéndose 268 encuestas íntegras.

\section{RESULTADOS}

CARACTERIZACIÓN DE LA MUESTRA

De la muestra final, $43 \%$ de los encuestados corresponden a mujeres y $57 \%$ a hombres, con un promedio de edad de 20 años y 8 meses. En términos socioeconómicos, 36\% pertenece al GSE alto, 37\% al medio alto, $19 \%$ al segmento medio y $3 \%$ al medio-bajo. Es decir, se trata de una muestra joven, con mayor presencia masculina, con educación universitaria y dominada por la presencia de personas pertenecientes a los segmentos altos y medio-alto (que totalizan $73 \%$ ).

En relación al auto posicionamiento político, se observa un valor promedio de 5,1 (5,5 es el medio de la escala), donde $20,6 \%$ se ubica en la izquierda (valores 1-3), 35,2\% en la centro izquierda (valores 4-5), $31,7 \%$ en la centro derecha (valores $6-7$ ) y $10,2 \%$ en la derecha (valores 8-10), lo que señala una muestra bastante equilibrada en términos políticos, con una leve predominancia del sector de izquierda (valores iguales o menores a 5) (gráfico 1).

\section{DIMENSIONALIDAD Y CONFIABILIDAD \\ DE LA ESCALA}

El análisis de componentes principales entregó una estructura de cinco factores con eigenvalues superiores a 1,0, explicando $63,87 \%$ de la varianza, medida de adecuación muestral de 0,959 y test de esfericidad de Barlett significativo $(0,000)$. Ningún ítem/rasgo fue eliminado, dado que en su totalidad poseen comunalidades que están muy cerca o sobre el valor 0,5 (tabla 2). 


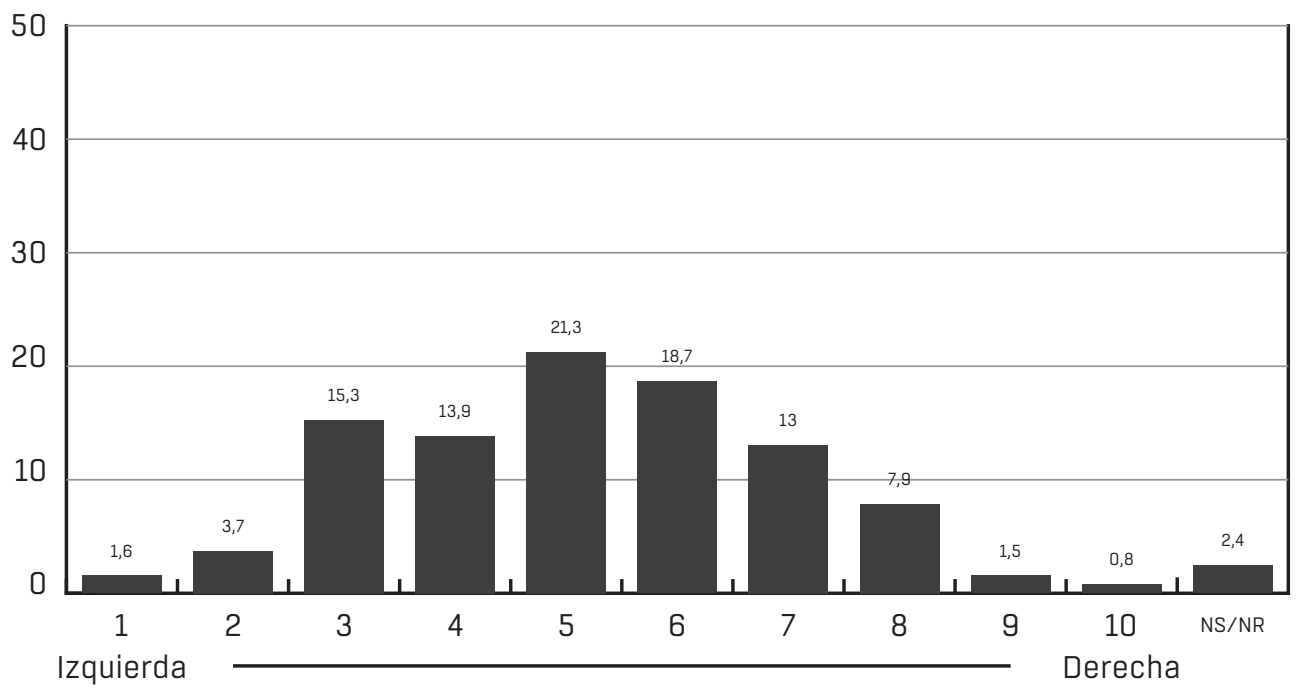

Gráfico 1. Distribución de la muestra por auto posicionamiento político [\%]

Fuente: Elaboración propia.

En esta estructura factorial, la primera dimensión fue denominada honesto/sincero, la cual posee $43,8 \%$ de varianza explicada, siendo la más importante. Está compuesta por seis rasgos: honesto, cercano, sincero, íntegro, real y confiable, y su nombre deriva de que el elemento central al que apuntan estos rasgos se refiere a que los partidos políticos tengan capacidad de mostrarse tal como son. La segunda dimensión, capacidad/ competencia, alcanza una varianza explicada de $7,0 \%$. Considera once rasgos: exitoso, profesional, inteligente, seguro, fuerte, líder, resistente, masculino, trabajador, tranquilo y realista, y se denominó de esta forma ya que la mayoría de los rasgos apuntan a tener mayores condiciones técnicas para poder llevar a la práctica las propuestas y gestionar un gobierno. La tercera dimensión fue denominada actual/contemporáneo, que posee una varianza explicada de 5,8\%. Consta de siete rasgos: actual, cool, a la moda, contemporáneo, joven, emocionante y atractivo, y fue denominada de esa forma debido a que todos los elementos que la componen apuntan a estar acorde a los tiempos actuales. La cuarta dimensión se denominó energético/vibrante, y explica 3,7\% de la varianza. Incluye los rasgos energético, osado, imaginativo, sentimental, amistoso y alegre, y se le dio ese nombre ya que todos los rasgos apuntan centralmente a tener carácter y decisión de llevar adelante cosas. Finalmente, la quinta dimensión fue denominada único/diferente, y explica 3,5\% de la varianza. Incorpora tres rasgos: único, independiente y original, y su nombre deriva de que los rasgos refieren a la capacidad de diferenciación del resto de los partidos (tabla 2).

Considerando la confiabilidad de los conjuntos de rasgos incorporados en cada una de las dimensiones de personalidad de marca, es posible observar que los indicadores de fiabilidad o Alfa de Cronbach de cada uno de ellos muestran niveles superiores a 0,7 (tabla 2). De esta forma, se puede señalar que la escala de medición de personalidad de marca posee adecuadas propiedades psicométricas en términos de dimensionalidad y confiabilidad en el contexto de los partidos políticos en Chile (Malhotra, 2008).

\section{PERCEPCIÓN DE LOS PARTIDOS POLITICOS}

A continuación, se procedió a examinar ${ }^{3}$ la percepción de personalidad de marca específica de los tres partidos incorporados en este estudio. Un primer resultado relevante de mencionar se refiere a que, a nivel de resultados totales (por dimensión, por partido y total general), la evaluación que existe de los partidos políticos en la muestra de jóvenes empleada en este estudio es bastante negativa. En el rango de 1 a 5 (ver metodología), se observa que el promedio general de los partidos evaluados en este estudio llega a 2,27, un número que señala que aquellos rasgos de personalidad de marca serían deseables para generar un buen vínculo con los partidos son escasos. Ello también se detecta al comparar las medias generales de los partidos, donde se aprecian cifras entre 2,17 y 2,41. Es importante mencionar que entre la Democracia Cristiana (DC) y la Unión 


\begin{tabular}{|c|c|c|c|c|c|c|c|c|}
\hline Dimensión & Rasgo & 1 & 2 & 3 & 4 & 5 & $\%$ Varianza & $\begin{array}{l}\text { Alpha de } \\
\text { Cronbach }\end{array}$ \\
\hline \multirow{6}{*}{ Honesto/Sincero } & Honesto & 0,81 & 0,12 & 0,24 & 0,16 & 0,19 & \multirow{6}{*}{43,79} & \multirow{6}{*}{0,92} \\
\hline & Cercano & 0,77 & 0,18 & 0,29 & 0,18 & 0,18 & & \\
\hline & Sincero & 0,73 & 0,18 & 0,26 & 0,22 & 0,24 & & \\
\hline & integro & 0,71 & 0,33 & 0,19 & 0,16 & 0,05 & & \\
\hline & Real & 0,66 & 0,24 & 0,27 & 0,25 & 0,22 & & \\
\hline & Confiable & 0,63 & 0,34 & 0,34 & 0,11 & 0,18 & & \\
\hline \multirow{11}{*}{$\begin{array}{l}\text { Capaz/ } \\
\text { Competente }\end{array}$} & Exitoso & 0,21 & 0,77 & 0,21 & 0,05 & 0,06 & \multirow{11}{*}{7,01} & \multirow{11}{*}{0,89} \\
\hline & Profesional & 0,39 & 0,70 & 0,16 & 0,18 & 0,08 & & \\
\hline & Inteligente & 0,29 & 0,69 & 0,25 & 0,20 & 0,05 & & \\
\hline & Seguro & 0,39 & 0,64 & 0,22 & 0,15 & 0,13 & & \\
\hline & Fuerte & 0,04 & 0,61 & 0,20 & 0,35 & 0,40 & & \\
\hline & Líder & 0,16 & 0,60 & 0,34 & 0,25 & 0,15 & & \\
\hline & Resistente & 0,02 & 0,54 & 0,13 & 0,46 & 0,42 & & \\
\hline & Masculino & $-0,05$ & 0,52 & $-0,13$ & 0,01 & 0,41 & & \\
\hline & Trabajador & 0,38 & 0,50 & 0,20 & 0,38 & 0,17 & & \\
\hline & Tranquilo & 0,45 & 0,49 & 0,03 & $-0,12$ & $-0,09$ & & \\
\hline & Realista & 0,46 & 0,48 & 0,36 & 0,07 & 0,06 & & \\
\hline \multirow{7}{*}{$\begin{array}{l}\text { Actual/ } \\
\text { contemporáneo }\end{array}$} & Actual & 0,21 & 0,27 & 0,74 & 0,18 & 0,11 & \multirow{7}{*}{5,81} & \multirow{7}{*}{0,90} \\
\hline & Cool & 0,34 & 0,10 & 0,70 & 0,20 & 0,20 & & \\
\hline & A la moda & 0,19 & 0,23 & 0,69 & 0,25 & $-0,01$ & & \\
\hline & Contemporáneo & 0,26 & 0,27 & 0,66 & 0,24 & 0,15 & & \\
\hline & Joven & 0,13 & 0,02 & 0,65 & 0,44 & 0,14 & & \\
\hline & Emocionante & 0,30 & 0,10 & 0,63 & 0,36 & 0,23 & & \\
\hline & Atractivo & 0,39 & 0,30 & 0,61 & 0,10 & 0,13 & & \\
\hline \multirow{7}{*}{$\begin{array}{l}\text { Energético/ } \\
\text { Vibrante }\end{array}$} & Animado & 0,15 & 0,20 & 0,20 & 0,76 & 0,10 & \multirow{7}{*}{3,73} & \multirow{7}{*}{0,83} \\
\hline & Osado & 0,01 & 0,04 & 0,26 & 0,74 & 0,12 & & \\
\hline & Imaginativo & 0,07 & 0,07 & 0,29 & 0,64 & 0,06 & & \\
\hline & Emocional & 0,29 & 0,10 & 0,04 & 0,64 & 0,07 & & \\
\hline & Amistoso & 0,48 & 0,22 & 0,21 & 0,53 & $-0,03$ & & \\
\hline & Alegre & 0,32 & 0,26 & 0,39 & 0,50 & $-0,02$ & & \\
\hline & Extrovertido & 0,16 & 0,33 & 0,31 & 0,49 & 0,36 & & \\
\hline \multirow{3}{*}{ Único/Diferente } & Único & 0,28 & 0,15 & 0,18 & 0,13 & 0,72 & \multirow{3}{*}{3,54} & \multirow{3}{*}{0,75} \\
\hline & Independiente & 0,34 & 0,16 & 0,27 & 0,11 & 0,61 & & \\
\hline & Original & 0,45 & 0,10 & 0,40 & 0,18 & 0,49 & & \\
\hline
\end{tabular}

Tabla 2. Tabla resumen con matriz de componentes rotados: rasgos personalidad de marca, dimedimensiones y confiabilidad

Fuente: Elaboración propia.

CUADERNOS.INFO N 41 / DICIEMBRE 2017 / ISSN 0719-3661 / Versión electrónica: www.cuadernos.info / ISSN 0719-367x 


\begin{tabular}{lllll} 
Dimensión & UDI & DC & PC & Total \\
\hline Honesto/Sincero & 1,97 & 2,19 & 2,17 & 2,11 \\
\hline Capaz/Competente & 2,71 & 2,44 & 2,52 & 2,56 \\
\hline Actual/Contemporáneo & 1,91 & 1,84 & 2,19 & 1,98 \\
\hline Energético/Vibrante & 2,34 & 2,36 & 2,88 & 2,53 \\
\hline Único/Diferente & 2,24 & 2,05 & 2,28 & 2,19 \\
\hline Total & 2,23 & 2,17 & 2,41 & 2,27 \\
\hline
\end{tabular}

Tabla 3. Evaluación de la personalidad de marca de los partidos políticos

Fuente: Elaboración propia.

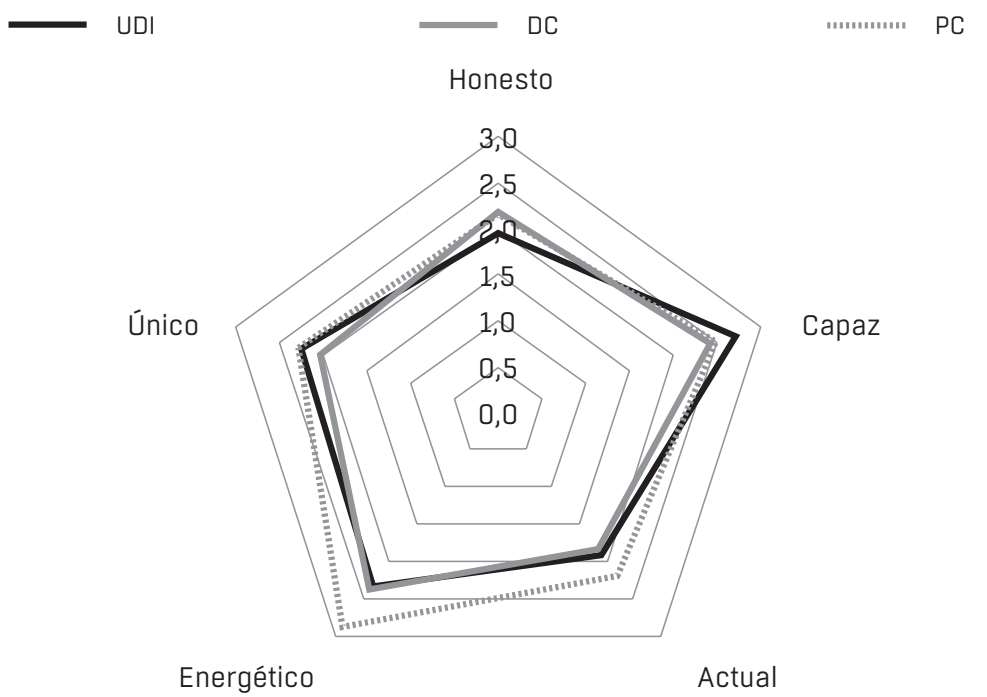

Gráfico 2. Personalidad de marca de los partidos políticos evaluados

Fuente: Elaboración propia.

Demócrata Independiente (UDI) no hay diferencias significativas en los resultados totales, no así entre el Partido Comunista (PC) con la DC $(t=3,590 ; p=0,000)$ y con la UDI $(t=2,558, p=0,011)$, donde el primero de estos partidos (PC) posee una evaluación ligera, pero significativamente, menos mala (tabla 3).

Mirando por dimensiones de personalidad de marca, el PC posee diferencias estadísticamente significativas con el resto de los partidos evaluados en varias de ellas (ver tabla 4). Una refiere a la honestidad (la variable que más explica la varianza), en donde la UDI posee un promedio más bajo que la DC $(t=2,783, p=0,006)$ y que el PC $(t=2,380, p=0,018)$. La segunda es en actual/contemporáneo, donde también el PC posee superioridad sobre la UDI $(t=3,603, p=0,000)$ y la DC $(t=4,577, p=0,000)$ La tercera corresponde a la dimensión denominada energético/vibrante, donde también el PC supera tanto a la UDI $(t=6,795, p=0,000)$ como a la DC $(t=6,690$, $p=0,000)$. Una situación opuesta se da en el caso de la aptitud/competencia, que es el atributo en que la UDI posee superioridad frente tanto a la DC $(t=3,542$, $p=0,000)$ como al PC $(t=2,425, p=0,016)$. Finalmente, en el caso de único/diferente, es la DC la que posee peor evaluación que la UDI $(t=2,116, p=0,035)$ y que el PC $(t=2,511, p=0,012)$. Así, se puede observar que el PC posee una evaluación menos negativa en tres atributos, destacando sobre todo su energía; la UDI -por su parte-es relativamente mejor evaluada en términos 
UDI

DC

PC

\begin{tabular}{lccc|ccc|ccc}
\hline & Alto & $\begin{array}{c}\text { Medio } \\
\text { alto }\end{array}$ & $\begin{array}{c}\text { Medio } \\
\text { bajo } \\
\text { y bajo }\end{array}$ & Alto & $\begin{array}{c}\text { Medio } \\
\text { alto }\end{array}$ & $\begin{array}{c}\text { Medio } \\
\text { bajo } \\
\text { y bajo }\end{array}$ & Alto & $\begin{array}{c}\text { Medio } \\
\text { alto }\end{array}$ & $\begin{array}{c}\text { Medio } \\
\text { bajo } \\
\text { y bajo }\end{array}$ \\
\hline Honesto/Sincero & 2,23 & 1,96 & 1,56 & 2,34 & 2,19 & 1,92 & 2,12 & 2,23 & 2,19 \\
\hline Capaz/Competente & 2,91 & 2,69 & 2,42 & 2,54 & 2,47 & 2,22 & 2,49 & 2,55 & 2,53 \\
\hline $\begin{array}{l}\text { Actual/ } \\
\text { Contemporáneo }\end{array}$ & 2,10 & 1,88 & 1,63 & 2,01 & 1,80 & 1,64 & 2,10 & 2,24 & 2,25 \\
\hline Energético/Vibrante & 2,50 & 2,32 & 2,13 & 2,56 & 2,32 & 2,08 & 2,87 & 2,87 & 2,92 \\
\hline Único/Diferente & 2,50 & 2,19 & 1,92 & 2,20 & 2,12 & 1,69 & 2,32 & 2,37 & 2,16 \\
\hline Total & 2,45 & 2,21 & 1,93 & 2,33 & 2,18 & 1,91 & 2,30 & 2,45 & 2,41 \\
\hline
\end{tabular}

Tabla 4. Personalidad de marca de los partidos políticos evaluados según grupo socioeconómico

Fuente: Elaboración propia.

\begin{tabular}{lccc|ccc|ccc} 
& \multicolumn{3}{c}{ Izquierda } & \multicolumn{4}{c}{ Centro } & \multicolumn{3}{c}{ Derecha } \\
& UDI & DC & PC & UDI & DC & PC & UDI & DC & PC \\
\hline Honesto/Sincero & 1,47 & 2,12 & 2,60 & 1,98 & 2,21 & 2,04 & 2,66 & 2,26 & 1,78 \\
\hline Capaz/Competente & 2,37 & 2,41 & 2,82 & 2,67 & 2,42 & 2,40 & 3,30 & 2,49 & 2,35 \\
\hline $\begin{array}{l}\text { Actual/ } \\
\text { Contemporáneo }\end{array}$ & 1,57 & 1,71 & 2,48 & 1,87 & 1,87 & 2,03 & 2,45 & 2,02 & 2,05 \\
\hline Energético/Vibrante & 2,11 & 2,18 & 3,02 & 2,36 & 2,35 & 2,84 & 2,65 & 2,64 & 2,81 \\
\hline Único/Diferente & 2,03 & 1,98 & 2,54 & 2,07 & 1,99 & 2,03 & 2,84 & 2,23 & 2,30 \\
\hline Total & 1,91 & 2,08 & 2,69 & 2,19 & 2,17 & 2,27 & 2,78 & 2,33 & 2,26 \\
\hline
\end{tabular}

Tabla 5. Personalidad de marca de los partidos políticos evaluados por posición política

Fuente: Elaboración propia.

de su aptitud (que es la segunda dimensión en relevancia), mientras que la DC no logra ser percibida con alguna superioridad relativa en ninguna dimensión.

\section{ANÁLISIS DE LOS PARTIDOS POLÍTICOS SEGÚN VARIABLES INDEPENDIENTES}

Alexaminar la percepción de la personalidad de marca por las variables sexo, GSE y auto-posicionamiento político, surgen algunas diferencias interesantes de mencionar. Con respecto al sexo, la prueba ANOVA muestra que las percepciones de personalidad de marca son similares en todos los factores. La única excepción es cuando se compara el caso específico de la dimensión honesto en el partido UDI, en el que las mujeres lo evalúan mejor que los hombres $(t=2,582, p=0,009)$.

Una situación diferente se observa por nivel socioeconómico. Al comparar el grupo alto (ABC1), con el medio-alto (C2) y el resto de los participantes (C3D), se aprecia que en el caso de la UDI existen diferencias significativas $(F=9,283, p=0,000)$, observándose mediante el contraste polinómico $(F=18.315$, $p=0,000)$ que este cambio es lineal: a medida que se desciende en la escala de GSE, empeora la evaluación general de este partido. Lo mismo ocurre con la DC, en la que se observan diferencias significativas respecto de su evaluación de personalidad de marca según $\operatorname{GSE}(F=7,113, p=0,000)$, diferencias que son lineales, tal como lo muestra el análisis polinómico $(F=13,754$, 


\begin{tabular}{|c|c|c|c|c|c|}
\hline $\begin{array}{c}\text { Aaker (1997) } \\
\text { EE.UU. } \\
42 \text { rasgos }\end{array}$ & $\begin{array}{l}\text { Varianza } \\
\text { explicada }\end{array}$ & $\begin{array}{c}\text { Smith (2009) Reino } \\
\text { Unido } \\
34 \text { rasgos }\end{array}$ & $\begin{array}{l}\text { Varianza } \\
\text { explicada }\end{array}$ & $\begin{array}{l}\text { Chile }[2017 \text { ] } \\
30 \text { rasgos }\end{array}$ & $\begin{array}{l}\text { Varianza } \\
\text { explicada }\end{array}$ \\
\hline $\begin{array}{l}\text { Realista, familiar, } \\
\text { cercano, honesto, } \\
\text { sincero, real, íntegro, } \\
\text { original, sentimental, } \\
\text { alegre y amistoso. }\end{array}$ & $26,5 \%$ & $\begin{array}{l}\text { Honesto, cercano, } \\
\text { integro, sincero, real, } \\
\text { sentimental, realista y } \\
\text { amistoso. }\end{array}$ & $32 \%$ & $\begin{array}{l}\text { Honesto, cercano, } \\
\text { sincero, íntegro, real y } \\
\text { confiable. }\end{array}$ & $43,8 \%$ \\
\hline $\begin{array}{l}\text { Osado, a la moda, cool, } \\
\text { independiente, único, } \\
\text { actual, emocionante, } \\
\text { imaginativo, animado, } \\
\text { contemporáneo y } \\
\text { joven. }\end{array}$ & $25,1 \%$ & $\begin{array}{l}\text { Tranquilo, atractivo, a } \\
\text { la moda, joven, cool, } \\
\text { emocionante y actual. }\end{array}$ & $6 \%$ & $\begin{array}{l}\text { Actual, cool, } \\
\text { contemporáneo, joven, } \\
\text { emocionante, a la } \\
\text { moda y atractivo. }\end{array}$ & $5,8 \%$ \\
\hline [No aplica] & - & $\begin{array}{l}\text { Animado, osado, } \\
\text { imaginativo, moderno } \\
\text { y alegre. }\end{array}$ & $10 \%$ & $\begin{array}{l}\text { Animado, osado, } \\
\text { imaginativo, } \\
\text { sentimental, amistoso, } \\
\text { alegre y extrovertido. }\end{array}$ & $3,7 \%$ \\
\hline $\begin{array}{l}\text { Confiable, trabajador, } \\
\text { seguro, inteligente, } \\
\text { profesional, líder, } \\
\text { empresarial, exitoso y } \\
\text { seguro de sí mismo. }\end{array}$ & $17,5 \%$ & $\begin{array}{l}\text { Líder, confiable, } \\
\text { inteligente, exitoso, } \\
\text { trabajador, profesional } \\
\text { y seguro. }\end{array}$ & $4 \%$ & $\begin{array}{l}\text { Exitoso, profesional, } \\
\text { inteligente, seguro, } \\
\text { fuerte, líder, } \\
\text { resistente, masculino, } \\
\text { trabajador, tranquilo y } \\
\text { realista. }\end{array}$ & $7,0 \%$ \\
\hline $\begin{array}{l}\text { Clase alta, glamoroso, } \\
\text { atractivo, encantador, } \\
\text { femenino y delicado. }\end{array}$ & $11,9 \%$ & [No aplica] & - & [No aplica] & - \\
\hline $\begin{array}{l}\text { Extrovertido, } \\
\text { masculino, occidental, } \\
\text { resistente y fuerte. }\end{array}$ & $8,8 \%$ & $\begin{array}{l}\text { Masculino, fuerte, } \\
\text { resistente y } \\
\text { extrovertido. }\end{array}$ & $5 \%$ & [No aplica] & - \\
\hline [No aplica] & - & $\begin{array}{l}\text { Único, original e } \\
\text { independiente. }\end{array}$ & $3 \%$ & $\begin{array}{l}\text { Único, independiente y } \\
\text { original. }\end{array}$ & $3,5 \%$ \\
\hline
\end{tabular}

Tabla 6. Comparación de las dimensiones de estudios de Aaker (1989), Smith [2009) y actual (agrupados por similitud relativa)

Fuente: Elaboración propia.

$p=0,000)$. Esto refleja en este caso que, a medida que se desciende en la escala de GSE, la evaluación de la DC también cae significativamente. Finalmente, en el PC se detecta una situación diferente, si bien descriptivamente su peor evaluación está en el segmento alto, la diferencias por GSE no resultan estadísticamente significativas (tabla 4).

Un último análisis realizado se refiere a la existencia de eventuales diferencias en la personalidad de marca de los partidos por posición política. Se agruparon agrupó los entrevistados en tres segmentos según auto posicionamiento: los valores 1 al 3 fueron etiquetados como izquierda, los valores 4 al 7 como centro y los valores 8 al 10 como derecha. Los resultados muestran que, entre las personas que se autodefinen como de izquierda y de derecha, existen diferencias significativas en la evaluación de los partidos políticos (tabla 5). En el caso de los de izquierda, a medida que la oferta de partidos se mueve hacia la izquierda, las personas los evalúan mejor $(F=28,851, p=0,000)$. En este caso, el análisis polinomial confirma que este cambio es lineal $(F=52,218, p=0,000)$. En el caso de los de derecha, existen diferencias $(F=8,376, p=0,000)$ que no son lineales en el continuo político. Muestran diferencias entre la evaluación de la UDI versus la DC $(t=3,253, p=0,001)$ y el PC $(t=3,599, p=0,000)$, pero no entre estos dos últimos 
partidos. Dicho de otro modo, las personas de derecha evalúan con mayor presencia de atributos de personalidad de marca a la UDI, y de modo similar a la DC y el PC.

\section{COMPARACIÓN DE RESULTADOS CON ESTUDIOS ANTERIORES}

Al comparar los resultados obtenidos en este estudio con los reportados por Smith (2009) en Inglaterra, así como el original de Aaker (1997), es posible observar que existen similitudes y diferencias importantes entre ellos (ver tabla 6)

En cuanto a las similitudes, la dimensión más importante en los tres estudios se relaciona con los elementos de honestidad/sinceridad. Aunque este rasgo se muestra en diversos estudios como el más relevante, en el caso de la política adquiere un particular poder explicativo, particularmente en Chile, transformándose - por lejosen el rasgo de personalidad de mayor importancia relativa. En segundo lugar, los tres estudios destacan una dimensión vinculada a ser actual/contemporáneo, así como otra vinculada a lo trabajador/profesional. Esos tres elementos aparecen transversalmente en los estudios utilizando tanto marcas comerciales como partidos de Inglaterra y Chile. Adicionalmente, estos dos estudios referidos a partidos políticos poseen alta similitud en términos de las dimensiones que se construyen con base en sus rasgos. Si bien en el estudio de Smith (2009) se presenta una dimensión relacionada con la dureza, en el resto de ellas son bastante similares más allá de la denominación que se haya escogido para cada factor. Esto sugiere la existencia de validez concurrente en los estudios de personalidad de marca de partidos políticos. A lo anterior hay que agregar que la varianza explicada por las dimensiones, en el caso de este estudio, es similar a la observada por Smith, que alcanza un $60 \%$ en las seis dimensiones con eigenvalue superior a 1 (este estudio logra un 63,87\%). Esto habla de que las dimensiones para evaluar los partidos tienden a ser, en esencia, similares entre los jóvenes universitarios de Reino Unido y de Chile, lo que da un fuerte sustento al uso del concepto de personalidad de marca en el ámbito de los partidos políticos.

En relación con las diferencias, aquellas más relevantes surgen al contrastar estos resultados con el estudio de Smith (2009), que posee seis factores, mientras que el estudio chileno solo cinco (tal como Aaker, 1997). Si bien esta estructura dimensional es similar en número, no es exactamente igual al comparar ambos estudios desarrollados en el contexto de partidos políticos. Además, estos dos estudios en personalidad de marca política poseen un nivel de varianza explicada en torno a 60\%, menor que la reportada por Aaker (1997), que logra sobre $80 \%$ en sus cinco dimensiones. Esto puede ser indicativo de que, si bien en el caso de los partidos políticos el modelo de personalidad de marca posee un buen nivel de poder explicativo, los rasgos de personalidad empleados son un menos potentes en el análisis de marcas políticas que en el análisis de aquellas en el mundo de los productos comerciales.

Por su parte, al comparar estos resultados con los observados por Araya y Etchebarne (2014b) en su análisis de la percepción de los partidos políticos, existen bastantes diferencias, dadas principalmente por la escasa comparabilidad de los estudios y por las limitaciones de cómo fueron reportados los datos por dichos autores. Por ejemplo, los partidos de derecha tendían a agruparse como conservadores y tradicionales, lo que no es concordante con la idea del atributo de capacidad más presente en estos siguiendo la clasificación de Aaker (1997) y Smith (2009).

\section{CONCLUSIONES}

Asumiendo que la construcción de una percepción positiva de un partido político es un elemento estratégico clave al que las diversas acciones comunicacionales apuntan, este estudio examinó la adecuación de la noción de personalidad de marca en este ámbito. Se aplicó la escala desarrollada originalmente por Aaker (1997) y adaptada a los partidos políticos por Smith (2009) en una muestra de jóvenes universitarios chilenos. Este grupo, como parte del segmento joven, representa un sector particularmente crítico y distante de la política actual, y al que diversos esfuerzos de la autoridad se han dirigido para fomentarles una mayor y mejor sintonía con el sistema democrático institucional.

Los resultados muestran, en primer lugar, la adecuación del modelo de personalidad de marca a la evaluación de los partidos políticos -al menos en Chile-, en el grupo de jóvenes universitarios. Existe una serie de dimensiones que subyace a las respuestas de las personas entrevistadas y que logra explicar casi dos tercios de la variabilidad en las respuestas obtenidas. Dicho de otro modo, es posible señalar que la medición de personalidad de marca en partidos políticos posee una buena validez de constructo, lo que permite plantear la pertinencia de los conceptos asumidos en su medición (Cronbach, 1960). Esto debe ser complementado con que los rasgos extraídos se vincularon sin problemas en un factor, cada uno con cargas factoriales en torno a 0,5 
o superiores (es decir, no hubo que eliminar ninguno en el estudio) y que la confiabilidad de las dimensiones se cumple, ya que los Alfas de Cronbach son superiores a 0,7 en todos los casos. Es decir, la medición y uso de la personalidad de marca en el contexto de los partidos políticos posee las propiedades psicométricas básicas necesarias para afirmar que estamos ante un concepto que da cuenta de forma adecuada del fenómeno que estamos estudiando.

A lo anterior se agrega que resultados similares en términos de dimensiones fueron observados en el Reino Unido anteriormente por Smith (2009). La similitud entre ambos estudios se encuentra a varios niveles: en cuanto a la estructura de factores, que difiere solo en uno (cinco versus seis); en relación al porcentaje de varianza explicada en ambos estudios por el listado de rasgos, que en ambos casos supera $60 \%$, y en términos de la similitud de los rasgos incorporados en cada dimensión. Deesta forma, existen elementos esenciales que hacen plantear la potencial existencia de validez concurrente del concepto de personalidad de marca en partidos políticos y del instrumento empleado para medirlo (Campbell \& Fiske, 1959; Messick, 1994).

\section{DISCUSIÓN}

Este estudio provee una herramienta metodológicamente sólida de evaluación estratégica de los partidos políticos en tanto entidades percibidas por la ciudadanía (es decir, como marcas). Los ciudadanos-los mismos que en otro contexto son consumidores- ven un modo de ser de los partidos (personalidad) y se vinculan en ese sentido con ellos en términos de su honestidad, competencia, ser actual, energía y ser diferente respecto de los demás (Scammell, 2015). Considerar estos elementos puede ayudar de modo significativo a los partidos a desarrollar y mantener relaciones positivas con la sociedad en general, y también a que quienes los componen sean más persuasivos (Ahmad \& Thyagaraj, 2015)

En ese sentido, esas características de personalidad, y en particular la primera (percepción de honestidad) aparecen como factores relevantes para generar una relación más poderosa entre la ciudadanía (al menos jóvenes universitarios) y el mundo político (Smith, 2009). Esto implica que debiese ser ese un eje fundamental de la estrategia de los partidos para mejorar su relación con la ciudadanía, y además da soporte a la acción actual de varios gobiernos de la región que están tomando medidas en términos de regular y transparentar la acción de los partidos, sobre todo en términos de su uso de recursos (Engel, 2015).

Lo anterior no significa que el uso del concepto de personalidad de marca explique toda la complejidad del problema. Este estudio muestra algunos elementos a considerar y que marcan alertas importantes de las limitaciones que posee. La primera de ellas es que las dimensiones detectadas en los estudios chilenos y británico con partidos políticos no son exactamente iguales y, que a su vez, estos también difieren parcialmente de los estudios realizados con marcas comerciales (Aaker, 1997; Olavarrieta et al., 2010).

A lo anterior se agrega que la varianza explicada en los estudios con marcas comerciales es superior a la que se ha podido observar en ya dos estudios con partidos políticos (Chile e Inglaterra). Ello muestra que el concepto de personalidad de marca, si bien funciona adecuadamente como herramienta estratégica de evaluación de los esfuerzos político-comunicacionales y posee los elementos básicos de validez y confiabilidad, requiere de ser usado criteriosamente. Es decir, que puede ser una herramienta relevante para examinary comprar la situación de los partidos políticos en Chile, pero que su proceso de importación desde el mundo de las marcas comerciales no está finalizado. Como señala Gorbaniuk et al. (2015), es posible que existan atributos adicionales a los señalados acá, propios de la política, que no estén considerados, los que deberían ser abordados por futuras investigaciones. Junto a ello, es importante medir la asociación efectiva entre el concepto de personalidad de marca y adhesión política y voto efectivo, para ver si ese aspecto que ha sido observado en el mercado de productos y servicios también se da en el contexto político (Ahmad \& Thyagaraj, 2015).

Junto a lo anterior, este estudio examinó de modo exploratorio la personalidad de marca de un set de partidos de diferentes tendencias, así como el rol de algunas variables como el sexo, el GSE y el auto posicionamiento político. Los resultados muestran, primero, que la evaluación general de los partidos es bastante negativa, observándose bajos puntajes en todas las dimensiones (escasa presencia de los elementos relevantes de personalidad de marca). Ello no hace más que confirmar la pobre imagen que existe en los jóvenes de los partidos políticos, como ha sido documentado por diversas investigaciones previas (Carrasco, 2010).

A nivel de diferencias en la percepción de la personalidad de marca de los partidos, es posible decir que, en un contexto general de mala evaluación, poseen algunas diferencias. El gran capital de la UDI es la 
idea de su aptitud/capacidad, lo cual es consistente con el discurso permanente de la derecha sobre eficacia y capacidad/competencia (Muñoz-Navarro, 2008). Ello se presenta en todos los segmentos socioeconómicos y posiciones políticas, aunque obviamente mejor entre las personas de nivel alto y de derecha. En el caso del PC, destaca en varios atributos por sobre los demás partidos (no siendo por ello una evaluación positiva), particularmente en la dimensión de energía. Ello estaría resaltando un aspecto de la izquierda y del PC, particularmente en el mundo universitario: ser activo, estar en las movilizaciones (Llamazarez \& Sandell, 2003). Además, este partido posee una evaluación relativamente similar en todos los segmentos socioeconómicos y el cambio en la percepción de su personalidad de marca se da más bien según posición política (peor evaluación en las personas de derecha). El caso de la DC ofrece una paradoja en este estudio. En una muestra en la que la gran mayoría de los entrevistados se auto declara de centro, los partidos con mejor evaluación relativa son la UDI (derecha) y el PC (izquierda). Esto estaría mostrando, aparentemente, la relevancia de tener algún elemento identificatorio del partido, como lo es en el caso del PC con la energía o de la UDI con la aptitud/competencia.

Finalmente, es importante destacar que la muestra utilizada (jóvenes universitarios) representa una limitación y una fortaleza del estudio. Una limitación por cuanto sus resultados poseen, al final del día, un carácter tentativo: se trata de una muestra no probabilística de jóvenes con alto nivel educacional y de nivel socioeconómico medio y alto. En ese sentido, futuros estudios deberían examinar otros segmentos para observar si los resultados detectados son también aquellos que se pueden obtener en grupos alternativos de mayor edad, diferente escolaridad y de sectores populares, de modo de que se posea mayor evidencia sobre las dimensiones clave de evaluación de los partidos políticos en Chilee, idealmente, en Latinoamérica (Valdez Cepeda, 2003).
Sin embargo, el hecho de que este estudio se haya realizado con jóvenes universitarios es también una fortaleza, pues permite examinar al menos en parte el caso del segmento juvenil. Como se puede apreciar, su evaluación de los partidos es deficiente, es decir, no posee ningún elemento destacado. Esto se observa en que no existen puntajes promedio sobre 3, que es el neutro en la escala de Likert empleada (incluso en algunos casos se reportan valores promedio cercanos a 1,9), lo que muestra que las dimensiones clave de personalidad de marca observadas en los partidos son débiles en este segmento joven y educado. Si se considera que la generación participante de este estudio ha mostrado una alta capacidad de movilización política en Chile (Cárdenas Neira, 2016), es notorio que se auto sitúe tan fuera de los espacios creados por los partidos, los que aparecen en este estudio como instancias con las cuales no parece relevante ni atractivo tener un vínculo.

Mirado desde una perspectiva más general, el estudio hace un llamado de atención sobre la relevancia de incorporar elementos de gestión de la comunicación e imagen desde otros ámbitos, como herramientas útiles para mejorar la efectividad de la comunicación política. En particular, es significativo el caso de estos elementos de análisis de la relación usuario-marca que describe el modelo de personalidad de marca de Aaker, que mostraron un nivel importante de explicación de la varianza cuando se pone como foco un partido (como si fuera una marca comercial)

Relacionado con esto, este estudio entrega evidencia de la aplicabilidad relativa del concepto de marca a los partidos políticos, como ha sido debatido por diversos autores (Marland, 2003). A la luz de los resultados, las personas (al menos los jóvenes) son capaces de percibir a estas entidades también en términos de los elementos que constituyen su relación con las marcas, lo cual es otra evidencia de que consumidor y ciudadano son roles distintos, pero comparten procesos comunes de evaluación de las entidades estudiadas (Smith, 2009).

NOTAS

1. Scientific Electronic Library Online (SciELO), que es una plataforma que incluye una colección seleccionada de publicaciones científicas latinoamericanas.

2. Para las elecciones presidenciales del año 2013, votaron alrededor de 5 millones 700 mil personas, de las cuales menos de 400.000 tenía entre 18 y 25 años (SERVEL, 2015).

3. Es importante aclarar en este punto que estas evaluaciones son solo de carácter exploratorio, ya que la muestra empleada no permite hacer extrapolaciones a un universo mayor de votantes.

4. En términos de estructura factorial, se comparan solo esos estudios ya que emplea el mismo set de ítems. 


\section{REFERENCIAS}

Aaker, J. (1997). Dimensions of brand personality. Journal of Marketing Research, 34(3), 347-356. https://doi.org/10.2307/3151897

Ahmad, A. \& Thyagaraj, K. (2015). Understanding the influence of brand personality on consumer behavior. Journal of Advanced Management Science, 3(1), 38-43. https://doi.org/10.12720/joams.3.1.38-43

Araya-Castillo, L. \& Etchebarne, S. (2014a). Personalidad de marca de partidos políticos: Una mirada desde la comunidad estudiantil universitaria [Brand personality of political parties: a glance from young university students]. Revista Latinoamericana de Ciencias Sociales, Niñez y Juventud, 12(1), 225 241. Retrieved from http://revistaumanizales.cinde.org.co/index.php/Revista-Latinoamericana/ article/view/1123

Araya-Castillo, L. \& Etchebarne, S. (2014b). Personalidad de marca de los partidos políticos: propuesta de modelo [Political Parties' Brand Personality: A suggested model]. Folios, (40), 67-89. https://doi.org/10.17227/01234870.40folios67.89

Austin, J., Siguaw, J., \& Mattila, A. (2003). A re-examination of the generalizability of the Aaker brand personality measurement framework. Journal of Strategic Marketing, 11(2), 77-92. https://doi.org/10.1080/0965254032000104469

Banerjee, S. \& Ray Chaudhuri, B. (2016). Influence of voter demographics and newspaper in shaping political party choice in India: An empirical investigation. Journal of Political Marketing, 1, 1-28. https://doi.org/10.1080/15377857.2016.1147513

Campbell, D. T. \& Fiske, D. W. (1959). Convergent and discriminant validation by the multitreatmultimethod matrix. Psychological Bulletin, 56(2), 81-105. https://doi.org/10.1037/h0046016

Cárdenas Neira, C. (2016). El movimiento estudiantil chileno (2006-2016) y el uso de la web social: nuevos repertorios de acción e interacción comunicativa [The Chilean student movement (20062016) and the use of the social web: new action and communicative interaction repertoires]. Ultima Década, 24(45), 93-116. https://doi.org/10.4067/S0718-22362016000200006

Carrasco, G. (2010). Participación y tendencias políticas en estudiantes universitarios: el caso de la Universidad de Chile [Political participation and trends in university students: the case of the University of Chile]. Ultima Década, 18(32), 85-103. https://doi.org/10.4067/S0718-22362010000100005

Cronbach, L. J. (1960). Essentials for Psychological Testing. New York: Harper and Row.

Engel, E. (2015). Informe Final: Consejo Asesor Presidencial contra los Conflictos de Interés, el Tráfico de Influencias y la Corrupción [Final Report: Presidential Advisory Council against Conflicts of Interest, Trafficking in Influences and Corruption]. Revista de derecho (Valdivia), 28(2), 267-269. https://doi.org/10.4067/S0718-09502015000200015

Farrandi, J., Valette-Florence, P., \& Fine-Falcy, S. (2000). Aaker's brand personality scale in a French context: A replication and preliminary test of validity. In H. E. Spotts, \& H. L. Meadow (Eds.), Developments of Marketing Science, Vol. 23. (pp. 7-13) Montreal: Academy of Marketing Science.

Frías, S. M. (2001). Opiniones y Actitudes (N³9) Cultura politica en España: Conocimiento, Actitudes, y Práctica [Opinion and Attitudes ( $\mathrm{N}^{\circ}$ 39) Political Culture in Spain: Knowledge, Attitudes and Practice]. Madrid: Centro de Investigaciones Sociológicas.

Gorbaniuk, O., Kusak, K., Kogut, A., \& Kustos, M. (2015). Dimensions of political party "personality" perception. Journal of Political Marketing, 14(1-2), 35-63. https://doi.org/10.1080/15377857.2014.990840

Guthrie, S. E. (1997). Anthropomorphism: A definition and a theory. In R.W. Mitchell, N.S. Thompson, \& H.L. Miles (Eds.), Antropomorphism Anecdots and Animals (pp.50-58). Albany, New York: State University of New York Press.

Kantar Ibope Media (2016). Establishment Survey 2016. Working paper. Santiago, Chile.

Keller, K. L. (2003). Brand synthesis: The multidimensionality of brand knowledge. Journal of Consumer Research, 29(4), 595-600. https://doi.org/10.1086/346254 
Lee, H. S. \& Cho, C. H. (2012). Sporting event personality: Scale development and sponsorship implications. International Journal of Sports Marketing and Sponsorship, 14(1), 46-63. https://doi.org/10.1108/IJSMS-14-01-2012-B005

Llamazares, I. \& Sandell, R. (2003). Partidos políticos y dimensiones ideológicas en Argentina, Chile, México y Uruguay. Esbozo de un análisis espacial [Political parties and ideological dimension in Argentina, Mexico and Uruguay: Outline of a spatial analysis]. Polis: Investigación y Análisis Sociopolitico y Psicosocial, (99), 43-70. Retrieved from http://polismexico.izt.uam.mx/index.php/rp/ article/view/434

Lock, A. \& Harris, P. (1996). Political marketing-vive la difference! European Journal of Marketing, 30(10/11), 14-24. https://doi.org/10.1108/03090569610149764

Malhotra, N. (2008). Investigación de Mercados [Marketing Research]. (5 ed.). Mexico: Pearson/Prentice Hall.

Marland, A. (2003). Marketing political soap: A political marketing view of selling candidates like soap, of electioneering as a ritual, and of electoral military analogies. Journal of Public Affairs, 3(2), 103-115. https://doi.org/10.1002/pa.139

Maureira, S. T. (2008). De lo épico a lo cotidiano: Jóvenes y generaciones políticas en Chile [From the Epic to the Everyday: Youth and Political Generations in Chile]. Revista de Ciencia Politica, 28(2), 143 160. https://doi.org/10.4067/S0718-090X2008000200006

Messick, S. (1994). Foundations of validity: Meaning and consequences in psychological assessment. European Journal of Psychological Assessment, 10(1), 1-9.

Muñoz-Navarro, A. (2008). La nación en el discurso de la derecha. Un análisis ideológico del debate parlamentario chileno [The concept of nation within de right-wing discourse. An ideological analysis in the Chilean Congress debate]. Signo y Pensamiento, 27(53). 174-191. Retrieved from http://www.scielo.org.co/scielo.php?script=sci_arttext\&pid=S0120-48232008000200011

Murphy, L., Moscardo, G., \& Benckendorff, P. (2007). Using brand personality to differentiate regional tourism destinations. Journal of Travel Research, 46(1), 5-14. https://doi.org/10.1177/0047287507302371

Newman, B. I. (1999). The Mass Marketing of Politics: Democracy in an Age of Manufactured Images. London: Sage Publications.

Olavarrieta, S., Friedmann, R., \& Manzur, E. (2010). Brand Personality in Chile: a combined emic-etic approach. Estudios de Administración, 17(1), 25-50. Retrieved from http://estudiosdeadministracion. unegocios.cl/images/stories/pdf/E_A_2010_VOL_17_N1_solavarrieta_rfriedmann_emanzur.pdf

O'Shaughnessy, N. J. (1990). The Phenomenon of Political Marketing. London: Springer.

Peng, N. \& Hackley, C. (2009). Are voters, consumers? A qualitative exploration of the voter-consumer analogy in political marketing. Qualitative Market Research: An International Journal, 12(2), 171-186. https://doi.org/10.1108/13522750910948770

Rothschild, M. L. (1979). Marketing communications in nonbusiness situations or why it's so hard to sell brotherhood like soap. The Journal of Marketing, 43(2), 11-20. https://doi.org/10.2307/1250737

Sandoval, J. \& Carvallo, V. (2017). Discursos sobre política y democracia de estudiantes universitarios chilenos de distintas organizaciones juveniles [Discourses on politics and democracy by Chilean students from different youth organizations]. Revista Española de Ciencia Política, (43), 137-160. https://doi.org/10.21308/recp.43.06

Scammell, M. (2015). Politics and image: the conceptual value of branding. Journal of Political Marketing, 14(1-2), 7-18. https://doi.org/10.1080/15377857.2014.990829

Schneider, H. (2004). Branding in politics—manifestations, relevance and identity-oriented management. Journal of Political Marketing, 3(3), 41-67. https://doi.org/10.1300/J199v03n03_03

SERVEL (2015). Resultados electorales [Electoral results]. Retrieved from http://www.servel.cl/ss/site/ elecciones_presidenciales_1989_al_2013.html 
Smith, G. (2009). Conceptualizing and testing brand personality in British politics. Journal of Political Marketing, 8(3), 209-232. https://doi.org/10.1080/15377850903044858

Valdez Zepeda, A. (2003). El debate contemporáneo sobre la mercadotecnia política en América Latina: Una respuesta a sus críticos [The contemporary debate on political marketing in Latin America: a response to its critics]. Revista Latina de Comunicación Social, 6(56). Retrieved from https://www.ull. es/publicaciones/latina/20035624valdez.htm

\section{SOBRE LOS AUTORES}

Rodrigo Uribe, psicólogo (Pontificia Universidad Católica de Chile), magíster en Comunicación Política y doctor en Comunicación (Universidad de Sheffield). Profesor asociado de la Facultad de Economía y Negocios de la Universidad de Chile. Director del Magíster en Marketing de la Universidad de Chile. Sus líneas de investigación son el estudio de audiencias, los efectos de la publicidad en adultos y niños, las nuevas formas de comunicación de marketing y el uso de redes sociales como medio persuasivo.

Cristian Buzeta, ingeniero comercial, magíster en Marketing (Universidad de Chile) y estudiante doctoral en Economía Aplicada (Universidad de Antwerpen). Investigador de la Facultad de Economía y Negocios de la Universidad de Chile. Ex gerente comercial y de atención a clientes en Kantar IBOPE Media - Chile y director de proyectos de investigación de mercado. Mantiene una línea de investigación en social media, marketing y audiencias. Es instructor adjunto de la Facultad de Comunicaciones de la Pontificia Universidad Católica de Chile y profesor a tiempo parcial de Investigación de Mercados en la Universidad de Chile.

Javiera Reyes, economista y magíster en Marketing (Universidad de Chile). Junto a su labor académica, ha sido dirigente estudiantil, llegando a ocupar la vicepresidencia de la Federación de Estudiantes de la Universidad de Chile (FECH). Sus intereses académicos son la comunicación política y los potenciales usos del marketing en el ámbito de la gestión de la comunicación política. 